\title{
Hiperparatiroidismo primário: caso clínico
}

Joana Tavares, ${ }^{1-2}$ Rui Nogueira, ${ }^{2}$ Flávio Campos Costa, ${ }^{3}$ Carlos Cruz ${ }^{4}$

\section{RESUMO}

Introdução: O diagnóstico de hiperparatiroidismo primário pode ocorrer de forma incidental em fases assintomáticas ou surgir tardiamente em contexto de queixas inespecíficas e prolongadas.

Descrição do caso: Doente de 32 anos, sexo feminino, sem antecedentes de relevo. Em fevereiro de 2012 sofre episódio de cólica renal severa, com necessidade de nefrostomia. A investigação complementar identificou litíase renal exuberante, com indicação para cateter duplo J preventivo. Em dezembro de 2012 sofre rutura do tendão do quadricípete após queda. Em março de 2013, nova queda por desequilíbrio com fratura do colo do úmero. Mantém queixas progressivas de lombalgias, dores ósseas, articulares e fraqueza muscular generalizadas, de características mecânicas, sem sinais inflamatórios, acabando por ficar dependente de cadeira de rodas em 2014. É referenciada ao hospital de referência por hipercalcemia grave e elevação da hormona paratiroideia (PTH 4300pg/ml). A ecografia cervical e cintigrafia das paratiroides confirmam suspeitas de hiperparatiroidismo primário por provável adenoma da paratiroide. Submetida a paratiroidectomia esquerda em maio de 2014, complicada por Síndroma do Osso Faminto, com evolução clínica e analítica favorável.

Comentário: O caso clínico procura alertar para o hiperparatiroidismo primário como uma hipótese de diagnóstico possível perante um quadro de queixas osteoarticulares inespecífico e arrastado. O médico de família deve estar alerta para este diagnóstico, tendo em conta a prevalência destas queixas na prática clínica e a importância do diagnóstico precoce.

Palavras-chave: Hiperparatiroidismo primário; Adenoma paratiroide; Hipercalcemia.

\section{INTRODUÇÃO}

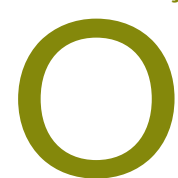

hiperparatiroidismo primário é uma entidade clínica caracterizada por um desequilíbrio no metabolismo do cálcio e fósforo, secundário a uma elevação da secreção da hormona paratiroideia (PTH). Esta patologia resulta predominantemente da presença de adenoma único, produtor de hormona paratiroideia, estando mais raramente associada a síndromas hereditárias ou tumores múltiplos da paratiroide..$^{-2}$

A incidência anual é de cerca de $0,2 \%$ nos pacientes acima dos 60 anos, tendo um pico de incidência entre a terceira e a quarta décadas. ${ }^{2}$

Verifica-se uma grande variabilidade na apresentação clínica, a qual está diretamente relacionada com a elevação da concentração plasmática de cálcio. A hipercalcemia ligeira (cálcio plasmático $<12 \mathrm{mg} / \mathrm{dL}$ ) pode

1. Médica Interna de Medicina Geral e Familiar

2. USF Norton de Matos

3. Centro Hospitalar e Universitário de Coimbra

4. IPOFG Coimbra ser assintomática ou apresentar-se sob a forma de sintomas inespecíficos, como obstipação, cansaço e depressão. A elevação da concentração plasmática de cálcio, sobretudo grave quando superior a $14 \mathrm{mg} / \mathrm{dL}$, pode causar sintomas marcados que incluem poliúria, polidipsia, desidratação, anorexia, fraqueza muscular. ${ }^{3-5}$

De um modo geral, a generalização das determinações de cálcio sérico leva a que muitos doentes sejam identificados em fases precoces e assintomáticas ou com sintomatologia inespecífica. No entanto, em casos mais graves, esta entidade pode cursar com uma grande diversidade de manifestações clínicas que incluem dores difusas, nefrolitíase ou extensa reabsorção óssea com fraturas (Quadro I). ${ }^{3-8}$

O diagnóstico é confirmado pela avaliação laboratorial com determinação dos valores de cálcio e PTH, sendo posteriormente feita avaliação ecográfica e cintigráfica da glândula. ${ }^{9}$

Quanto ao tratamento, na generalidade dos casos verifica-se reversão do quadro clínico com o tratamento cirúrgico, que consiste na excisão parcial ou total da glândula. ${ }^{10}$ 
QUADRO I. Manifestações clínicas da hipercalcemia

\section{MANIFESTAÇÕES CLÍNICAS DA HIPERCALCEMIA}

\begin{tabular}{|c|c|}
\hline $\begin{array}{l}\text { Distúrbios } \\
\text { neuropsiquiátricos }\end{array}$ & $\begin{array}{l}\text { Mais comuns: ansiedade, depressão e disfunção cognitiva } \\
\text { Mais graves: letargia, confusão, estupor }\end{array}$ \\
\hline $\begin{array}{l}\text { Distúrbios } \\
\text { gastrointestinais }\end{array}$ & $\begin{array}{l}\text { Obstipação } \\
\text { Anorexia } \\
\text { Náuseas } \\
\text { Pancreatites } \\
\text { Úlceras pépticas }\end{array}$ \\
\hline Disfunção renal & $\begin{array}{l}\text { Poliúria } \\
\text { Nefrolitíase } \\
\text { Hipercalciúria } \\
\text { Nefrocalcinose } \\
\text { Insuficiência renal aguda e crónica }\end{array}$ \\
\hline $\begin{array}{l}\text { Doença } \\
\text { cardiovascular }\end{array}$ & $\begin{array}{l}\text { HTA } \\
\text { Miocardiopatia } \\
\text { Calcificação valvular e vascular } \\
\text { Arritmias (casos de hipercalcemia grave: encurtamento do intervalo QT, elevação segmento ST) }\end{array}$ \\
\hline $\begin{array}{l}\text { Sintomas } \\
\text { músculo-esqueléticos }\end{array}$ & $\begin{array}{l}\text { Fraqueza muscular } \\
\text { Dores ósseas } \\
\text { Fraturas (redução do osso cortical, redução da densidade mineral óssea) }\end{array}$ \\
\hline
\end{tabular}

Os autores pretendem, com este trabalho, descrever um caso clínico relativo a uma patologia frequente, mas com uma apresentação grave e rara, resultante de um atraso no diagnóstico. Pretende-se salientar de forma resumida algumas das manifestações clínicas mais frequentes, métodos de diagnóstico e tratamento. É ainda objetivo primordial alertar os médicos de cuidados de saúde primários para a possibilidade do diagnóstico de hiperparatiroidismo primário perante queixas muito comuns na prática clínica diária.

\section{DESCRIÇÃO DO CASO}

Doente de 32 anos, sexo feminino, raça caucasiana, natural da Batalha, estudante universitária. Pertencente a uma família nuclear com três elementos, em fase VI do ciclo de Duvall e escala de Graffar classe III. Sem antecedentes pessoais de relevo. Sem hábitos nocivos. Antecedentes familiares irrelevantes. Sem medicação habitual.

Em fevereiro de 2012 sofre episódio de cólica renal severa, com necessidade de realização de nefrostomia por obstrução do ureter, seguida de fragmentação ul- trassónica do cálculo. A investigação complementar identificou presença de litíase renal exuberante, pelo que foi orientada para colocação de cateter duplo J com objetivo preventivo. Neste contexto realizou quatro sessões de litotrícia pouco efetivas.

Em dezembro de 2012 sofre rutura do tendão do quadricípete após queda, com indicação para repouso e analgesia. Em março de 2013 sofre nova queda por desequilíbrio com fratura do colo do úmero. Sem indicação para intervenção cirúrgica, tem recuperação prolongada, com queixas de lombalgias persistentes, dores ósseas e articulares generalizadas, de características mecânicas, sem sinais inflamatórios. Em abril de 2014, agravamento das queixas de dores ósseas e de fraqueza muscular generalizada, acabando por ficar dependente de cadeira de rodas. Durante este período, a doente recorre a vários ortopedistas, sendo apenas acompanhada de forma mais regular pelo nefrologista. Por exaustão sintomática recorre ao médico assistente, onde é realizado estudo analítico, ecografia cervical e renal, bem como osteodensitometria e radiografia dos joelhos e bacia. 

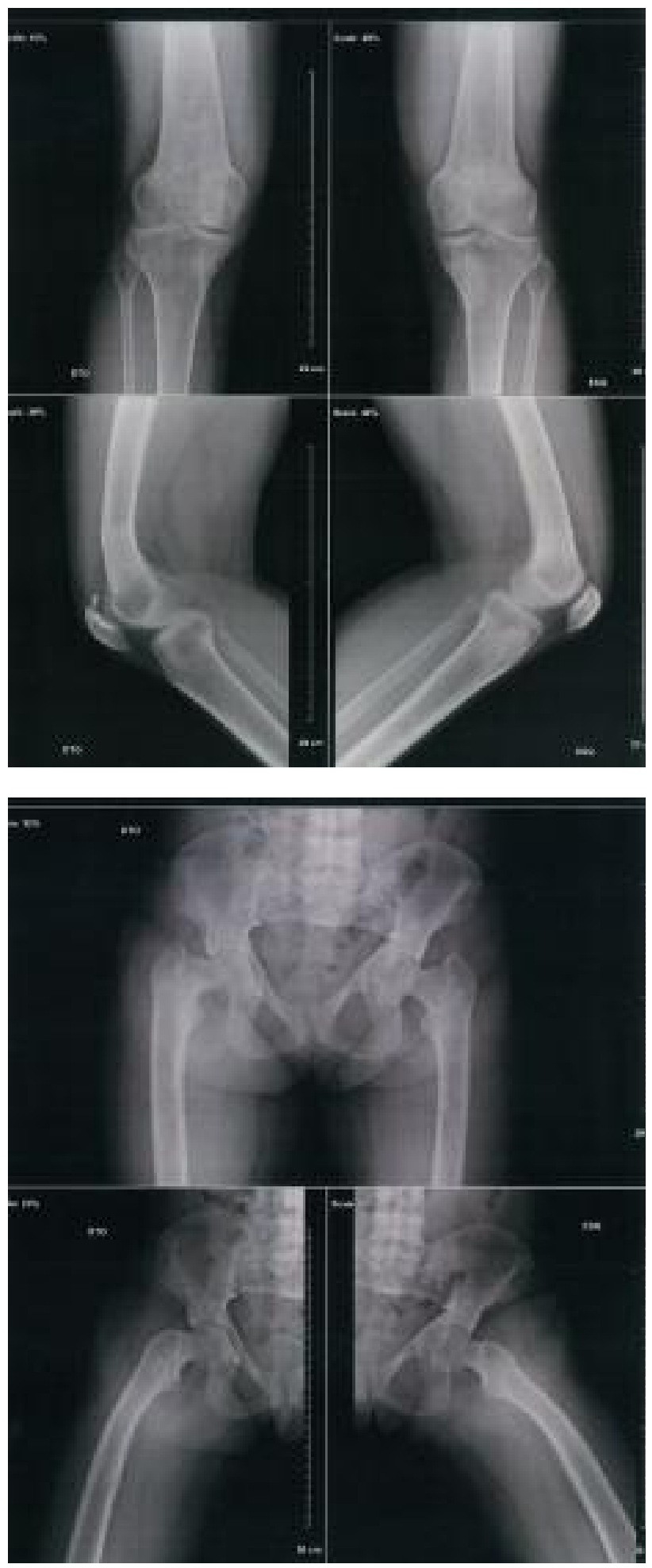

Figuras 1 e 2. Rarefação óssea generalizada (radiografia dos joelhos e bacia).
Analiticamente apresenta hipercalcemia (Ca2+ total $16,8 \mathrm{mg} / \mathrm{dl}, \mathrm{Ca} 2+$ ionizado de $3,95 \mathrm{mg} / \mathrm{dl}$ ), hipofosfatemia $(0,8 \mathrm{mg} / \mathrm{dl})$, elevação acentuada da fosfatase alcalina (3087 U/l), bem como elevação marcada da hormona paratiroideia (PTH $4300 \mathrm{pg} / \mathrm{ml}$ ) e calciúria $(12,22 \mathrm{mmol} / 24 \mathrm{~h})$. Sem alterações do hemograma e função tiroideia normal. A ecografia cervical revela "uma formação hipoecogénica com 18mm de maior eixo, vascularizada, podendo corresponder a glândula paratiroideia com dimensões aumentadas".

A ecografia renal revela "uma redução da espessura do parênquima e perda da diferenciação parênquimocentral do rim direito, com dilatação do sistema pielocalicial, observando-se cateter duplo J no interior e imagem sugestiva de foco litiásico na transição do bacinete para o grupo calicial superior com cerca de nove milímetros. No terço inferior do rim esquerdo, presença de foco litiásico com nove milímetros no grupo calicial inferior, sem dilatação significativa do sistema excretor".

A utente apresenta ainda uma osteodensitometria que demonstra osteoporose grave (T score de -6,1 na coluna lombar e -6,9 no fémur), com densidade mineral óssea muito abaixo do normal para a idade e sexo.

A radiografia dos joelhos revela rarefação óssea, sobretudo em localização medular, a nível das extremidades distal do fémur e proximal da tíbia, bem como imagens osteolíticas sugestivas de calcificação em vários pontos do esqueleto (Figura 1). A radiografia da bacia revela deformidade da extremidade proximal de ambos os fémures, verificando-se bilateralmente um ângulo de praticamente $90^{\circ}$ entre o colo do fémur e a diáfise femoral, com ligeiro estreitamento do colo do fémur bilateralmente (Figura 2).

A doente é referenciada para o hospital de referência pelo médico assistente por suspeita de hiperparatiroidismo primário.

A nível hospitalar realiza cintigrafia das paratiroides que é sugestiva de adenoma da paratiroide inferior esquerda. Realiza ainda TC cervico-toraco-abdominal: "Adjacente à vertente posterior do pólo inferior do lobo esquerdo da tiroide, observa-se uma formação nodular sólida com 16mm compatível com lesão da paratiroide, muito provavelmente adenoma (...) Identificadas extensas alterações estruturais ósseas que julgamos relação com informação clínica de hiperparatiroidismo". 
Colocada como hipótese de diagnóstico um hiperparatiroidismo primário por adenoma da paratiroide.

Após realização de estudos para exclusão de MEN tipo II, a doente é submetida a paratiroidectomia esquerda em maio de 2014. O exame histológico confirma a hipótese de adenoma da glândula paratiroide esquerda com dois centímetros.

De salientar que o pós-operatório complica-se por queixas de parestesias nos membros inferiores, superiores e periorais associadas a uma Síndroma do Osso Faminto (Hungry-bone Syndrome).

A evolução clínica é favorável, com melhoria progressiva das queixas álgicas. A doente tem alta com indicação para realização de fisioterapia e reavaliação periódica para controlo sintomático e analítico. Recupera mobilidade com apoio de canadianas em julho de 2014. Mantém atual seguimento em consulta de endocrinologia e urologia, estando medicada com citrato de potássio 1080mg id, nitrofurantoína 100mg id, vitamina D e cálcio.

O Quadro II permite observar a evolução analítica entre a primeira avaliação realizada em ambulatório pelo médico assistente e a última avaliação em consulta de cirurgia no hospital de referência. De salientar a melhoria acentuada dos valores analíticos que se correlacionam com a melhoria clínica da utente.

\section{COMENTÁRIO}

A propósito deste caso abordam-se alguns pontos de reflexão.

Por um lado, é de salientar que o diagnóstico clínico de hiperparatiroidismo primário pode ser um desafio, dada a natureza inespecífica dos sintomas. Tal dificuldade pode atrasar o diagnóstico, sendo este caso um exemplo de um diagnóstico muito tardio.

Por um lado, a idade jovem da utente, a presença de litíase renal bilateral grave, fraturas ósseas múltiplas e fraqueza muscular sem causa aparente deveriam ter suscitado a investigação desta hipótese de diagnóstico mais precocemente. Por outro lado, por si só, a presença de fratura de fragilidade associada a história de quedas numa doente jovem deveria ter suscitado investi- gação imediata de causas de osteoporose secundária.

Apesar do diagnóstico tardio, a excisão cirúrgica do adenoma, como causa para o hiperparatiroidismo, permitiu a reversão do quadro clínico com controlo analítico e clínico. ${ }^{9-10}$

Apesar da reversibilidade de alguns dos sintomas, são de realçar as consequências a nível renal e ortopédico: a desdiferenciação cortico-medular, a redução de espessura parenquimatosa evidente em ecografia, bem como a osteoporose e a fratura óssea decorrida. Tratamse de efeitos irreversíveis com necessidade de cuidados perenes e orientação em consulta especializada.

De realçar que o pós-operatório foi complicado por hipocalcemia grave e sintomática. Esta situação resultou de um hipoparatiroidismo pós-cirúrgico, denominado Síndroma do Osso Faminto (Hungry-bone Syndrome). Este quadro resulta de um estado de remineralização extensa do esqueleto, com necessidade de suplementação com cálcio e vitamina D por períodos prolongados. ${ }^{11}$

Merece ainda maior reflexão o atraso no diagnóstico em causa. Trata-se de uma utente não frequentadora dos cuidados de saúde primários, tendo recorrido a vários serviços de urgência e médicos de entidades particulares. A ausência de uma visão holística levou a um atraso considerável no diagnóstico, possível razão pela qual se verificaram manifestações tão exuberantes.

Em suma, este caso procura alertar o médico de família, pela sua posição privilegiada e visão abrangente, para o conhecimento do hiperparatiroidismo primário como possibilidade diagnóstica perante um 
quadro arrastado de queixas osteoarticulares inespecíficas.

\section{REFERÊNCIAS BIBLIOGRÁFICAS}

1. Potts Jr JT, Jüppner H. Disorders of the parathyroid gland and calcium homeostasis. In: Longo DL, Fauci AS, Kasper DL, Hauser SL, Jameson JL, Loscalzo J, editors. Harrison's principles of internal medicine. 18th ed. McGraw-Hill; 2011. p. 2592-602. ISBN 9780071748896

2. Marcocci C, Saponaro F. Epidemiology, pathogenesis of primary hyperparathyroidism: current data. Ann Endocrinol (Paris). 2015;76(2): 113-5.

3. Machenahalli P, Shotliff K. Problem based review: the patient with hypercalcaemia. Acute Med. 2015;14(3):138-41.

4. Fuleihan GE, Silverberg SJ. Primary hyperparathyroidism: clinical manifestations, diagnosis, differential diagnosis, and evaluation. UpToDate [homepage]; 2015 Apr [updated 2017 Nov 20]. Available from: https://www.uptodate.com/contents/primary-hyperparathyroidismdiagnosis-differential-diagnosis-and-evaluation/print

5. Vestergaard P. Primary hyperparathyroidism and nephrolithiasis. Ann Endocrinol (Paris). 2015;76(2):116-9.

6. Bandeira F, Cusano NE, Silva BC, Cassibba S, Almeida CB, Machado VC, et al. Bone disease in primary hyperparathyroidism. Arq Bras Endocrinol Metabol. 2014;58(5):553-61.

7. Bandeira F, Cassibba S. Hyperparathyroidism and bone health. Curr
Rheumatol Rep. 2015;17(7):48.

8. Nieciecki M, Cacko M, Królicki L.The role of ultrasound and nuclear medicine methods in the preoperative diagnostics of primary hyperparathyroidism. J Ultrason. 2015;15(63):398-409.

9. Lumachi F, Basso SM. Pathophysiology and treatment of nonfamilial hyperparathyroidism. Recent Pat CNS Drug Discov. 2014;9(3):164-72.

10. Roizen J, Levine MA. A meta-analysis comparing the biochemistry of primary hyperparathyroidism in youths to the biochemistry of primary hyperparathyroidism in adults. J Clin Endocrinol Metab. 2014;99(12): 4555-64.

11. Berkoben M, Quarles LD. Hungry bone syndrome following parathyroidectomy. UpToDate [homepage]; 2012 Nov [updated 2018 Jan 17]. Available from: https://www.uptodate.com/contents/hungry-bonesyndrome-following-parathyroidectomy

\section{CONFLITO DE INTERESSES}

Os autores declaram não ter quaisquer conflitos de interesse.

\section{ENDEREÇO PARA CORRESPONDÊNCIA \\ Joana Tavares \\ E-mail: joanaf.a.tavares@gmail.com \\ http://orcid.org/0000-0002-5038-0326}

Recebido em 27-01-2017

Aceite para publicação em 08-05-2018

\section{ABSTRACT}

\section{PRIMARY HYPERPARATHYROIDISM: A CASE REPORT}

Introduction: The diagnosis of primary hyperparathyroidism may occur incidentally in asymptomatic phases or appear late in the context of nonspecific and prolonged complaints.

Case report: We describe the case of a 32-year-old female without relevant previous medical history. In February 2012, she suffered an episode of severe renal colic, requiring nephrostomy. Further investigation identified exuberant renal lithiasis, with indication for a preventive double J catheter. In December 2012, the patient had a rupture of the quadriceps tendon due to a fall, and in March 2013, had a new fall due to imbalance with a resulting fracture of the humeral neck. The patient maintained progressive complaints of back pain, bone and joint pain and generalised muscle weakness, and eventually became wheelchairdependent in 2014. The patient was referred to the hospital for severe hypercalcemia and raised parathyroid hormone (PTH $4300 \mathrm{pg} / \mathrm{ml}$ ). Cervical ultrasound and parathyroid scintigraphy confirmed the clinical suspicion of primary hyperparathyroidism due to a parathyroid adenoma. Left parathyroidectomy was performed in May 2014, complicated with a Hungry-Bone syndrome. Clinical and analytical evolution was favorable.

Comment: This clinical case seeks to alert to primary hyperparathyroidism as a differential diagnosis when facing non-specific and prolonged osteoarticular complaints. Due to the prevalence of these complaints in clinical practice and the importance of an early diagnosis, the family physician should be alert to this potential diagnosis.

Keywords: Primary hyperparathyroidism; Parathyroid adenoma; Hypercalcemia. 